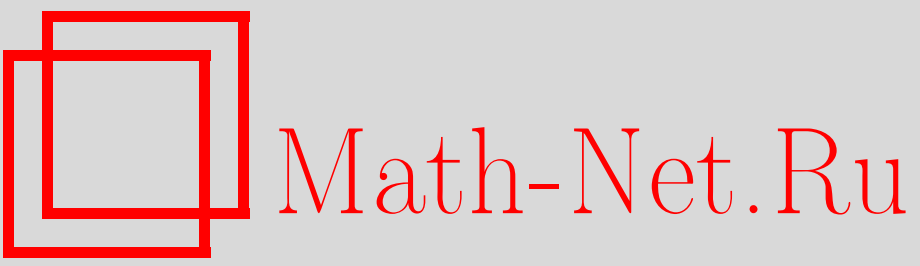

В. В. Дубровский, А. Н. Типко, З. С. Чекашкина, Регуляризованные следы унитарных операторов, УМH, 2001, том 56, выпуск 6, 145-146

DOI: https://doi.org/10.4213/rm459

Использование Общероссийского математического портала Math-Net.Ru подразумевает, что вы прочитали и согласны с пользовательским соглашением

http://www.mathnet.ru/rus/agreement

Параметры загрузки:

IP: 54.197 .130 .99

26 апреля 2023 г., 13:43:26 


\title{
РЕГУЛЯРИЗОВАННЫЕ СЛЕДЫ УНИТАРНЫХ ОПЕРАТОРОВ
}

\author{
В. В. ДУБРОвСКИй, А. Н. ТИПкО, З. С. ЧЕКАШКИНА
}

В этой работе, исползуя преобразование Кэли самосопряженных операторов (см. [1]), будет вьгислен регуляризованньй след унитарного оператора. Наш резултат не следует из статьи М. Г. Крейна [2].

Пусть $V_{i}$ - унитарные операторы в сепарабельном гильбертовом пространстве $H$ со спектром, состоящим из собственных значений $\beta_{n}^{\imath}$ конечной кратности, сходящихся к точке 1 , причем вьполнено условие Кэли: множество всех векторов $V_{i} h-h, h \in H$, плотно в $H, i=1,2$.

Введем самосопряженные операторы $A_{i}, i=1,2$, с помощью обратного оператора к преобразованию Кэли: $A_{i}=\left(z E-\bar{z} V_{i}\right)\left(1-V_{i}\right)^{-1}$. Предположим, что оператор $A_{1}$ имеет ядерную резольвенту или, что то же самое:

$$
\sum_{n=-\infty}^{\infty}\left|\beta_{n}^{1}-1\right|<\infty
$$

где $\beta_{n}^{i}$ - собственные числа оператора $V_{i}$, занумерованные согласно формуле

$$
\lambda_{n}^{i}=\left(z E-\beta_{n}^{i}\right)\left(1-\beta_{n}^{i}\right)^{-1},
$$

причем собственные числа $\lambda_{n}^{i}$ оператора $A_{i}$ занумерованы целыми числами в порядке возрастания величин с учетом кратности и $\lim _{n \rightarrow \infty} \lambda_{n}^{i}= \pm \infty, i=1,2$ (операторы $A_{i}$ могут быть полуограниченными). Будем дополнительно предполагать, что оператор

$$
A_{2}-A_{1}=\left(z E-\bar{z} V_{2}\right)\left(1-V_{2}\right)^{-1}-\left(z E-\bar{z} V_{1}\right)\left(1-V_{1}\right)^{-1}
$$

продолжается по непрерьвности до ограниченного, всюду определенного оператора в $H$; очевидно, что $\lim _{n \rightarrow \pm \infty} \beta_{n}^{\imath}=1, i=1,2$.

По лемме из [3] существуют две последовательности положительных чисел $\left\{a_{m}^{+}\right\}_{m=1}^{\infty}$ и $\left\{a_{m}^{-}\right\}_{m=1}^{\infty}$ такие, что

$$
\lim _{m \rightarrow \infty}\left\{\sum_{n=0}^{\infty} \frac{1}{\left|\lambda_{n}^{1}-a_{m}^{+}\right|}+\sum_{n=1}^{\infty} \frac{1}{\left|\lambda_{-n}^{1}+a_{m}^{-}\right|}\right\}=0 .
$$

Имеем

$$
\begin{aligned}
\sum_{n=-k_{m}}^{n_{m}}\left(\beta_{n}^{2}-\beta_{n}^{1}\right) & =-\frac{1}{2 \pi i} \operatorname{Sp} \int_{\Gamma_{m}} \frac{\lambda-z}{\lambda-\bar{z}}\left(\left(A_{2}-\lambda E\right)^{-1}-\left(A_{1}-\lambda E\right)^{-1}\right) d \lambda \\
& =-\frac{1}{2 \pi i} \operatorname{Sp} \int_{\Gamma_{m}} \frac{\lambda-z}{\lambda-\bar{z}} \sum_{t=1}^{\infty}\left(\left(A_{1}-\lambda E\right)^{-1}\left[\left(A_{2}-A_{1}\right)\left(A_{1}-\lambda E\right)^{-1}\right]^{t}\right) d \lambda
\end{aligned}
$$

Здесь $\Gamma_{m}$ - конечный замкнутьй регулярньй контур, содержащий на вещественной оси внутри себя толшко отрезок $\left[-a_{m}^{-}, a_{m}^{+}\right]$и отстоящий от вещественной оси на расстоянии не меньше чем $d_{m}=\min _{t=\overline{0, \infty}}\left\{\left|\lambda_{t}^{1}-a_{m}^{+}\right|,\left|\lambda_{-t}^{1}+a_{m}^{-}\right|\right\}$. Целше числа $\left(-k_{m}, n_{m}\right)$ суть номера ближайших на отрезке $\left[-a_{m}^{-}, a_{m}^{+}\right]$собственных чисел оператора $A_{1}$ от чисел $-a_{m}^{-}, a_{m}^{+}$соответственно.

Работа первого автора выполнена при поддержке Российского фонда фундаментальных исследований. 
Из (3) следует, что $d_{m}>\infty$ при $m \rightarrow \infty$. Оценим в ядерной норме на вертикальных прямых $\operatorname{Re} \lambda=-a_{m}^{-}$и $\operatorname{Re} \lambda=a_{m}^{+}$выражение $S(\lambda)=\sum_{t=2}^{\infty}\left(A_{1}-\lambda E\right)^{-1}\left[\left(A_{2}-A_{1}\right)\left(A_{1}-\lambda E\right)^{-1}\right]^{t}$ :

$$
\|S(\lambda)\|_{1} \leqslant\left\{\sum_{n=0}^{\infty} \frac{1}{\left|\lambda_{n}^{1}-a_{m}^{+}\right|}+\sum_{n=1}^{\infty} \frac{1}{\left|\lambda_{-n}^{1}+a_{m}^{-}\right|}\right\} * \frac{\left(\left\|A_{2}-A_{1}\right\|\left(d_{m}+|\rho|\right)^{-1}\right)^{2}}{1-\left\|A_{2}-A_{1}\right\|\left(d_{m}+|\rho|\right)^{-1}},
$$

где $\rho=\operatorname{Im} \lambda$. Тогда

$$
\mathrm{Sp} \int_{\Gamma_{m}} \frac{\lambda-z}{\lambda-\bar{z}} S(\lambda) d \lambda \rightarrow 0 \text { при } m \rightarrow \infty .
$$

С помощью теории вычетов вычислим слагаемые в (4):

$$
\begin{aligned}
\sum_{n=-\infty}^{\infty}\left(\beta_{n}^{2}-\beta_{n}^{1}\right)= & \sum_{n=-\infty}^{\infty}\left(\left[\left(\left(z E-\bar{z} V_{2}\right)\left(1-V_{2}\right)^{-1} f_{n}, f_{n}\right)\right.\right. \\
& \left.\left.-\left(\left(z E-\bar{z} V_{1}\right)\left(1-V_{1}\right)^{-1} f_{n}, f_{n}\right)\right] \frac{-2 \operatorname{Im} z\left(1-\beta_{n}^{1}\right)^{2}}{\left(2 \operatorname{Im} z-\beta_{n}^{1}(\bar{z}+1)\right)^{2}}\right) \\
& +\sum_{n=-\infty}^{\infty} \frac{-2 \operatorname{Im} z(1-z)\left(\beta_{n}^{1}-\beta_{n}^{2}\right)}{\left(2 \operatorname{Im} z+\beta_{n}^{1}(\bar{z}-1)\right)\left(2 \operatorname{Im} z+\beta_{n}^{2}(\bar{z}-1)\right)} .
\end{aligned}
$$

Итак, доказана следующая

Теорема. Если $V_{i}$ - унитарные операторы со спектром, состоящим из собственных чисел конечной кратности, сходящихся $\mathcal{k} 1$, удовлетворяющие условию Кәли, ограничению (1), и оператор (2) ограничен, то верно соотношение

$$
\begin{aligned}
\sum_{n=-\infty}^{\infty}\left(\beta_{n}^{2}-\beta_{n}^{1}\right)= & \sum_{n=-\infty}^{\infty}\left(\left[\left(\left(z E-\bar{z} V_{2}\right)\left(1-V_{2}\right)^{-1} f_{n}, f_{n}\right)\right.\right. \\
& \left.\left.-\left(\left(z E-\bar{z} V_{1}\right)\left(1-V_{1}\right)^{-1} f_{n}, f_{n}\right)\right] \frac{-2 \operatorname{Im} z\left(1-\beta_{n}^{1}\right)^{2}}{\left(2 \operatorname{Im} z-\beta_{n}^{1}(\bar{z}+1)\right)^{2}}\right) \\
& +\sum_{n=-\infty}^{\infty} \frac{-2 \operatorname{Im} z(1-z)\left(\beta_{n}^{1}-\beta_{n}^{2}\right)}{\left(2 \operatorname{Im} z+\beta_{n}^{1}(\bar{z}-1)\right)\left(2 \operatorname{Im} z+\beta_{n}^{2}(\bar{z}-1)\right)},
\end{aligned}
$$

где все ряды сходятся абсолютно.

ЗАмечАниЕ. Этим методом можно вычислить регуляризованные следы всех натуральных порядков унитарного оператора $V_{2}$.

\section{СПИСОК ЛИТЕРАТУРЫ}

[1] М. А. Наймарк. Линейные дифференциальные операторы. М.: Наука, 1969. [2] М. Г. Крейн // J. Operator Theory. 1987. Т. 17. С. 129-187. [3] В. А. Садовничий, В. А. Конягин, С. В. Подольский // Докл. РАН. 2000. Т. 373. №1. С. 26-28. 